\title{
Androgen-independent LNCaP cells are a subline of LNCaP cells with a more aggressive phenotype and androgen suppresses their growth by inducing cell cycle arrest at the G1 phase
}

\author{
PAN YU, XIUZHI DUAN, YUE CHENG, CHUNHUA LIU, YUHUA CHEN, \\ WEIWEI LIU, BINBIN YIN, XUCHU WANG and ZHIHUA TAO
}

Department of Laboratory Medicine, The Second Affiliated Hospital of Zhejiang University

School of Medicine, Hangzhou, Zhejiang 310009, P.R. China

Received September 11, 2015; Accepted August 17, 2017

DOI: 10.3892/ijmm.2017.3125

\begin{abstract}
Androgen deprivation therapy (ADT, surgical or chemical castration) is the mainstay treatment for metastatic prostate cancer $(\mathrm{PCa})$; however, patients ineluctably relapse despite extremely low androgen levels. This evolution of PCa indicates its lethal progression. In this study, to mimic the traits of clinical PCa progression in vitro, we investigated the alterations in the cell biological characteristics in androgenindependent LNCaP cells (LNCaP-AI cells) compared with $\mathrm{LNCaP}$ cells. We also examined the effects of androgen on LNCaP and LNCaP-AI cell proliferation, androgen receptor (AR) expression and prostate-specific antigen (PSA) secretion. Furthermore, AR was silenced in the LNCaP and LNCaP-AI cells to detect the roles taht AR plays in cell growth, apoptosis and PSA secretion. We found that prolonged androgen ablation increased the LNCaP-AI cell growth rate and cell invasiveness, and induced epithelial-mesenchymal transition in the LNCaP-AI cells. Moreover, despite the fact that the LNCaP and LNCaP-AI cells expressed equal amounts of AR protein, androgen induced a greater secretion of PSA in the LNCaP-AI cells than in the LNCaP cells. The proliferation of the LNCaP-AI cells was not dependent on, but was suppressed by androgen, which led to arrest at the G1 phase. Conversely, androgen significantly increased $\mathrm{LNCaP}$ cell proliferation by promoting the G1-S transition. Moreover, the silencing of AR suppressed LNCaP and LNCaP-AI cell growth by inducing cell cycle arrest at the G1 phase rather than promoting apoptosis, and reduced PSA secretion. On the whole, our data suggest that LNCaP-AI cells have a more more aggressive phenotype compared with the LNCaP cells; AR remains a critical factor in
\end{abstract}

Correspondence to: Professor Zhihua Tao, Department of Laboratory Medicine, The Second Affiliated Hospital of Zhejiang University School of Medicine, Hangzhou, Zhejiang 310009, P.R. China

E-mail: zrtzh@zju.edu.cn

Key words: androgen-independent LNCaP cells, growth suppression, cell cycle arrest, androgen, androgen receptor the LNCaP-AI cells, and androgen suppresses LNCaP-AI cell growth by blocking the cell cycle at the G1 phase.

\section{Introduction}

Androgen deprivation therapy (ADT, surgical or chemical castration) has been the primary treatment for prostate cancer (PCa) used to suppress the transcriptional activity of androgen receptor (AR) since the 1940s; however, a proportion of patients relapse within a median of 2-3 years with disease that is usually more aggressive and is currently defined as castration-resistant PCa (CRPC) (1-3). It was well known that $\mathrm{AR}$ is actively involved in the development, progression and metastasis of PCa (4). Over the past several years, studies from many groups have proposed that AR is aberrantly re-activated in CRPC (4-6). There are multiple mechanisms through which AR is aberrantly re-activated to support cell growth in CRPC in the androgen-depleted condition, including AR amplification (the hypersensitive pathway), AR mutation and co-regulator alterations (the promiscuous pathway), ligand-independent AR activation (the outlaw pathway) (4).

In general, androgen is a driver which elicits $\mathrm{PCa}$ initiation and progression (7). However, it is contradictory that androgen plays a role as a suppressor in LNCaP cell growth by inducing cell cycle arrest at the G1 phase following treatment with high-dose dihydrotestosterone (DHT), accompanied by p27 overexpression and the loss of the phosphorylated retinoblastoma protein $(8,9)$. Intermittent androgen deprivation therapy (IADT) had been proposed for many years, and preclinical models have demonstrated that exposure to intermittent androgen leads to a delay in the time to castration resistance in castrated animals bearing androgen-dependent Shionogi carcinoma tumors (10). Nonetheless, in phase III clinical trials, there is still insufficient evidence to support that IADT prevents the long-term complications caused by ADT (11). Thus, the roles which androgen plays in PCa are closely dependent on the context.

LNCaP was considered as a universally used cell line derived from a human lymph node metastatic lesion of prostatic adenocarcinoma (12). Previously, we established a PCa progression model using LNCaP cells which were continuously maintained in androgen-depleted conditions to mimic patients subjected to 
androgen ablation therapy (13), terned the LNCaP-AI cell line. LNCaP-AI cells have been previously reported (14); however, there are few studies which completely explain the differences among the two cell lines. In this study, we examined the alterations in cell biological characteristics between the LNCaP and LNCaP-AI cells. We also investigated the roles which androgen and AR play in regulating $\mathrm{LNCaP}$ and LNCaP-AI cell growth, making a comparison between the LNCaP and LNCaP cells as regards AR expression and PSA secretion when androgen is absent or present. These findings shed more light into the role of androgen in $\mathrm{PCa}$ and enhance our understanding of $\mathrm{PCa}$ development and progression.

\section{Materials and methods}

Reagents and antibodies. Dulbecco's modified Eagle's medium (DMEM), phenol red-free DMEM, fetal bovine serum (FBS), charcoal-stripped FBS (CS-FBS), penicillin, streptomycin, $0.25 \%$ trypsin and phosphate-buffered saline (PBS) were purchased from Gibco (Gaithersburg, MD, USA). DHT with a purity of $>98 \%$, was purchased from Sigma-Aldrich (St. Louis, MO, USA). For all androgen regulation experiments, the cells were maintained in phenol red-free DMEM medium with $10 \%$ CS-FBS. The androgen DHT powder was dissolved in ethanol and diluted into the required concentrations with the culture medium. Antibodies against cyclin D1 (1:1,000; ab134175), Cdk4 (1:1,000; ab199728), Cdk2 (1:1,000; ab32147), retinoblastoma protein ( $\mathrm{pRb} ; 1: 1,000$; Ab173289) were purchased from Abcam (Cambridge, MA, USA). Antibodies against AR (1:1,000; 3202S), p21 (1:1,000; 2947S), p27 (1:1,000; 3686S), cyclin E1 (1:1,000; 4129S), E-cadherin (1:1,000; 14472S), $\mathrm{N}$-cadherin $(1: 1,000 ; 14215 \mathrm{~S})$ and glyceraldehyde 3-phosphate dehydrogenase (GAPDH; 1:1000; 5174S) were purchased from Cell Signaling Technology (Beverly, MA, USA).

Cells and cell culture. LNCaP cells obtained from the Cell Bank of Shanghai Institute of Biochemistry and Cell Biology, Chinese Academy of Sciences (Shanghai, China) were cultured in a $5 \% \mathrm{CO}_{2}$ incubator in DMEM with $10 \%$ FBS. The establishment of an androgen-independent cell line (LNCaP-AI) was carried out as previously described (13). The LNCaP-AI cells were maintained in phenol red-free DMEM with $10 \% \mathrm{CS}-\mathrm{FBS}$.

Cell proliferation assay. Cell proliferation was measured using a cell counting kit-8 (CCK-8; Dojindo Molecular Technologies, Kumamoto, Japan) following the manufacturer's instructions. Briefly, the cells were seeded in 96-well plate at a density of $5 \times 10^{3}$ cells/well. After $24 \mathrm{~h}$, they were treated with $10 \mathrm{nM}$ DHT or were transfected with AR short hairpin RNA (shRNA) and scrambled shRNA recombinant lentiviral vector for different periods of time. A total of $10 \mu \mathrm{lCCK}-8$ solution was added to each well of the plate and incubated at $37^{\circ} \mathrm{C}$ for $2 \mathrm{~h}$ in a humidified $5 \% \mathrm{CO}_{2}$ atmosphere. Cell viability was then determined by testing the absorbance at a $450 \mathrm{~nm}$ wavelength with a microplate reader (iMark ${ }^{\mathrm{TM}}$; serial no. 13492; Bio-Rad, Tokyo, Japan).

Transwell invasion assay. Transwells of 24-well coated with Matrigel (8- $\mu \mathrm{m}$ pore size; BD Biosciences, Bedford, MA, USA) were used in the cell invasion assay. The cells were cultured in serum-free medium overnight before being trypsinized and resuspended at a density of $5 \times 10^{4}$ cells $/ \mathrm{ml}$ in serum-free medium. A total of $1 \times 10^{4}$ cells $(200 \mu \mathrm{l})$ were seeded into the upper chambers. Subsequently, the lower chambers were supplemented with $500 \mu \mathrm{l}$ complete medium containing 10\% FBS as a chemoattractant. After $48 \mathrm{~h}$ of incubation, the Matrigel and the cells remaining in the upper chamber were removed using cotton swabs. The invaded cells were fixed in $4 \%$ paraformaldehyde and stained with $0.5 \%$ crystal violet, counted using an inverted microscope (Leica, Wetzlar, Germany).

Apoptosis analysis. Cell apoptosis was measured using the Annexin V-PE/7-AAD kit (BD Biosciences, San Diego, CA, USA). In brief, the cells were seeded in 6-well plates at a density of $2 \times 10^{5}$ cells/well and transfected with AR shRNA or scrambled shRNA recombinant lentivirus vector for $72 \mathrm{~h}$. The cells were typsinized and resuspended in $100 \mu 11 \mathrm{X}$ binding buffer, followed by the addition of $5 \mu \mathrm{l}$ of PE Annexin $\mathrm{V}$ and $5 \mu \mathrm{l}$ 7-AAD, and gently vortexing the cells and incubating for $15 \mathrm{~min}$ at room temperature $\left(25^{\circ} \mathrm{C}\right)$ in the dark. Subsequently, $400 \mu 11 \mathrm{X}$ binding buffer were replenished to each tube. Samples were analyzed using a flow cytometer (FACSCalibur; BD Biosciences, San Jose, CA, USA) within $1 \mathrm{~h}$ and CellQuest software (FACSCalibur).

Cell cycle analysis. Cell cycle distribution was measured by flow cytometry with PI/RNase staining buffer (BD Biosciences). In brief, $2 \times 10^{5}$ cells were exposed to ethanol (vehicle) or $10 \mathrm{nM}$ DHT and transfected with AR shRNA or scrambled shRNA recombinant lentivirus vector. The cells were then trypsinized, resuspended and fixed with $70 \%$ ethanol/30\% PBS at $-20^{\circ} \mathrm{C}$ overnight. The cells were then stained with PI/RNase staining buffer for $15 \mathrm{~min}$ in the dark and analyzed using a flow cytometer and ModFit LT software (FACSCalibur). For each measurement, $3 \times 10^{4}$ cells were collected and analyzed.

Transfection with shRNA lentiviral particles. shRNA lentiviral particles were purchased from GenePharma Biotechnology, Inc. (Shanghai, China). The sequence of the AR shRNA was as follows: 5'-GGAACTCGATCGTATCATTGC-3'; a scrambled sequence was used as a negative control with the following squence: 5'-TTCTCCGAACGT GTCACGT-3'. According to the instructions provided with the lentiviral particles, the cells were seeded at $2 \times 10^{5}$ cells/well in a 6 -well plate prior to lentiviral particles infection and incubated with $2 \mathrm{ml}$ of complete medium for $24 \mathrm{~h}$. The cells were infected by the addition of the shRNA lentiviral particles at a multiplicity of infection (MOI) of 50; the plates were then gently swirled for mixing followed by incubation for $12 \mathrm{~h}$. The virus-containing medium of infected wells was removed and fresh complete medium was added. The transfection efficiency was evaluated using a FACSCalibur (BD Biosciences) flow cytometer detecting green fluorescence protein (GFP) expression. Reverse transcriptionquantitative PCR (RT-qPCR) and western blot analysis were performed to determine the shRNA interference efficiency.

$R T-q P C R$. Total RNA was isolated using TRIzol reagent (Invitrogen, Carlsbad, CA, USA) and dissolved in RNAase-free water according to the manufacturer's instructions. Total RNA $(1.0 \mu \mathrm{g})$ was used as a template for cDNA synthesis using the PrimeScript $^{\mathrm{TM}}$ RT reagent kit with gDNA Eraser (Takara, Dalian, China). Real-time PCR was performed using FastStart 
Universal SYBR-Green Master (Roche, Indianapolis, IN, USA). The GAPDH mRNA level was used as an internal control. The sequences of primers were used as follows: AR forward, 5'-GCCACTCAGACCCACTTAGC-3' and reverse, 5'-CCTCA CTCTTCGTCCACATCG-3'; PSA forward, 5'-GCCCACTGC ATCAGGAACAA-3' and reverse, 5'-GCTGTGGCTGACCTG AAATACC-3'; GAPDH forward, 5'-AACAGCCTCAAGATC ATCAGCA-3' and reverse, 5'-CATGAGTCCTTCCACGATA CCA-3'. The reactions were performed using the ABI 7500 Real-Time PCR system (Applied Biosystems, Foster City, CA, USA). All data for each sample were collected in triplicate and assessed using $2^{-\Delta \Delta \mathrm{Cq}}$ relative quantitative analysis.

Determination of PSA secretion. The cells were seeded in a 6-well plate with androgen-depleted medium at a density of $3 \times 10^{5}$ cells/well and then treated with ethanol (vehicle) or $10 \mathrm{nM}$ DHT for $24 \mathrm{~h}$, and were transfected with AR shRNA or scrambled shRNA recombinant lentivirus vector for $72 \mathrm{~h}$. The cell culture supernatants were collected to measure the total PSA level on a chemiluminescence apparatus (Architect i2000SR; Abbott Diagnostics, Abbott Park, IL, USA) according to the manufacturer's instructions by chemiluminescence immunoassay (CLIA).

Western blot analysis. The cells were lysed in pre-cold RIPA lysis buffer containing protease inhibitor cocktail (Biotool, Houston, TX, USA), and placed sample on ice vigorously vortex $15 \mathrm{sec}$ every $10 \mathrm{~min}$ for 3 times. Cell lysates were centrifuged at $13,000 \mathrm{x}$ g for $10 \mathrm{~min}$ and the supernatants were collected. Protein concentrations were measured using the Pierce BCA protein assay kit (Pierce, Rockford, IL, USA) according to the manufacturer's instructions. Total protein $(40 \mu \mathrm{g})$ was loaded and separated by electrophoresis on $10 \%$ SDS-PAGE at $100 \mathrm{~V}$ for $2 \mathrm{~h}$ and transferred onto $0.2 \mu \mathrm{m}$ polyvinylidene difluoride membranes (Millipore, Billerica, MA, USA) using wet blotter at $250 \mathrm{~mA}$ for $2 \mathrm{~h}$. The membranes were blocked with $5 \%$ defatted milk for $1 \mathrm{~h}$ at room temperature and then incubated with primary antibody diluted in $5 \%$ bovine serum albumin at $4{ }^{\circ} \mathrm{C}$ overnight. After being washed 3 times for 15 min with $10 \%$ TBST, the membranes were incubated with HRP-conjugated secondary antibodies [anti-mouse IgG $(1: 5,000 ; 7076 \mathrm{~S})$ and anti-rabbit IgG (1:5,000; 7074S); both from Cell Signaling Technology] for $2 \mathrm{~h}$ at room temperature. The bound antibodies were visualized using an enhanced chemiluminescence kit (Millipore).

Statistical analysis. All data are presented as the means \pm standard deviation. Two-tailed Student's t-test was used and a value of $\mathrm{P}<0.05$ was considered to indicate a statistically significant difference. All statistical analyses were performed using SPSS 13.0 software (SPSS Inc., Chicago, IL, USA).

\section{Results}

Prolonged androgen deprivation increases the LNCAP-AI cell proliferation rate and invasiveness, and induces epithelialmesenchymal transition (EMT). To examine the alterations in the cell biological characteristics in the transition from LNCaP to LNCaP-AI cells, we performed CCK-8 cell proliferation assay, cell invasion assay and western blot analysis of EMT marker proteins in the LNCaP-AI cells and LNCaP cells. CCK-8 proliferation assay revealed that the LNCaP-AI cells grew adequately more rapidly than the LNCaP cells in androgen-depleted medium (Fig. 1A). In the LNCaP-AI cells, western blot analysis demonstrated a significant decrease in E-cadherin protein levels and a notable increase in $\mathrm{N}$-cadherin protein levels compared with the LNCaP cells, suggesting that EMT had occurred in the transition from LNCaP to LNCaP-AI cells (Fig. 1B). In addition, the numbers of invasive LNCaP-AI cells significantly increased compared with the LNCaP cells, demonstrating that prolonged androgen deprivation enhanced the LNCaP-AI cell invasive ability (Fig. 1C).

Higher AR transcriptional activity in the LNCAP-AI cells compared with the LNCaP cells. To examine the AR expression levels in the LNCaP-AI cells and compare them to those of the LNCaP cells, and to determine the effects of androgen on AR expression, western blot analyses were performed using the LNCaP and LNCaP-AI cells following treatment with ethanol (vehicle) or $10 \mathrm{nM}$ DHT. The results revealed that similar levels of AR protein were expressed in the LNCaP and LNCaP-AI cells, and neither the LNCaP nor LNCaP-AI cells expressed higher levels of AR protein following stimulation with DHT (Fig. 2A). We then examined the androgenic induction of mRNA expression and protein secretion of PSA, one of the AR target genes, in order to assess the transcriptional activity of AR in the LNCaP and LNCaP-AI cells. The PSA mRNA and protein levels markedly increased with DHT treatment in the LNCaP and LNCaP-AI cells. However, when DHT was absent or present, the PSA protein level in the LNCaP-AI cells was higher than that in the LNCaP cells (Fig. 2B and C). These findings demonstrated that the LNCaP-AI cells retained a similar level of AR as the LNCaP cells and were still sensitive to androgen stimulation, and that the expression of AR are not affected by androgen stimulation. The PSA protein levels in the LNCaP-AI cells were significantly higher than those in the LNCaP cells in the absence or presence of DHT.

Androgen suppresses the proliferation of LNCAP-AI cells by inducing cell cycle arrest at the G1 phase, contrary to the LNCaP cells. To determine the role that androgen plays in LNCaP-AI cell growth compared with the LNCaP cells, the LNCaP-AI and LNCaP cells were treated with DHT and cell viability was examined. We found that the proliferation of the LNCaP-AI cells was significantly suppressed by androgen $(10 \mathrm{nM}$ DHT) at a concentration that is appropriate for LNCaP cell growth (Fig. 3A). Subsequently, we performed flow cytometry to analyze the effects of androgen on the cell cycle distribution of the LNCaP-AI and LNCaP cells. The results demonstrated that androgen induced LNCaP-AI cell cycle arrest at the G1 phase, but promoted LNCaP cell G1-S transition (Fig. 3B). To investigate the underlying mechanisms, we measured the expression of G1 phase cell cycle-related proteins. The results of western blot analysis following treatment with $10 \mathrm{nM}$ DHT revealed the loss of the phosphorylated $\mathrm{pRb}(\mathrm{p}-\mathrm{pRb})$ and $\mathrm{Cdk} 2$ protein in the LNCaP-AI cells, in contrast to the LNCaP cells. This finding was in line with the DHT-mediated arrest in the G1 phase in the LNCaP-AI cells. There was no visible change in the protein levels of Cdk4, cyclin D1 and cyclin E1. In addition, DHT induced the upregulation of p27 and the downregulation of p21 in the LNCaP-AI cells, inconsistent with the fact that 
A

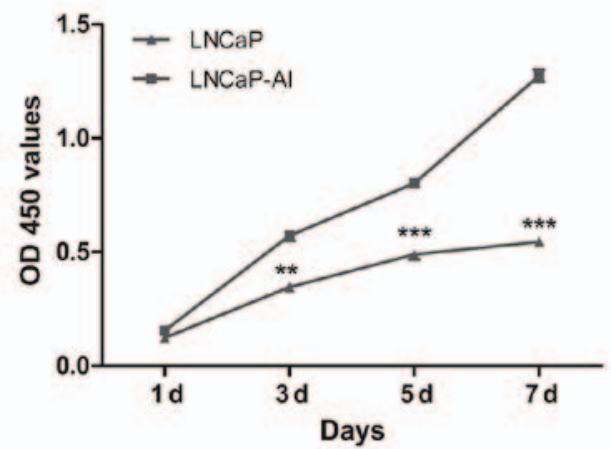

B

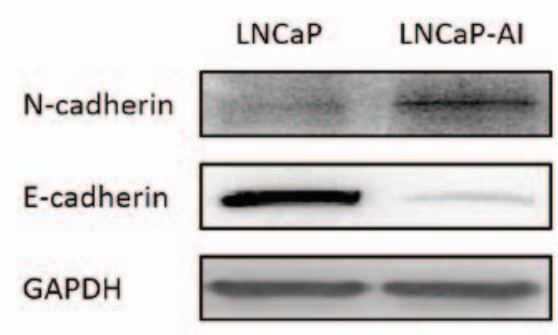

C

LNCaP

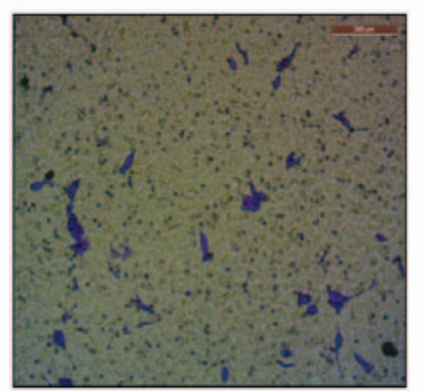

LNCaP-AI

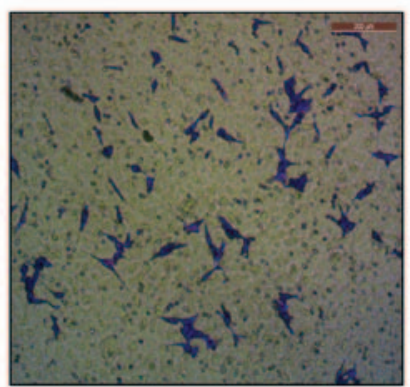

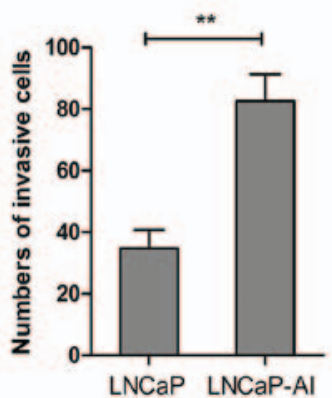

Figure 1. LNCaP-AI cells represent a more advanced disease phenotype. (A) LNCaP-AI cells grew more rapidly than the LNCaP cells in the absence of androgen. Cell proliferation was determined by cell counting kit-8 (CCK-8) assay. (B) Western blot analysis of E-cadherin and N-cadherin protein levels in the LNCaP and LNCaP-AI cells; GAPDH was used as a loading control. (C) Invasiveness of LNCaP and LNCaP-AI cells measured by Transwell invasion assay; the number of invaded LNCaP-AI cells significantly increased in the compared to the LNCaP cells (magnification, x200). Bars represent the means \pm SD of 3 replicates; ${ }^{* *} \mathrm{p}<0.01$ and ${ }^{* * *} \mathrm{p}<0.001$.

A

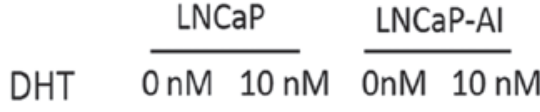

\section{AR}

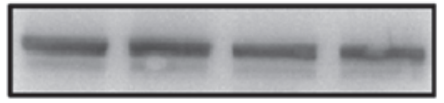

GAPDH

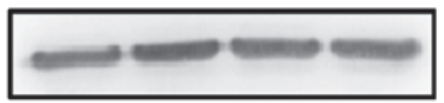

B

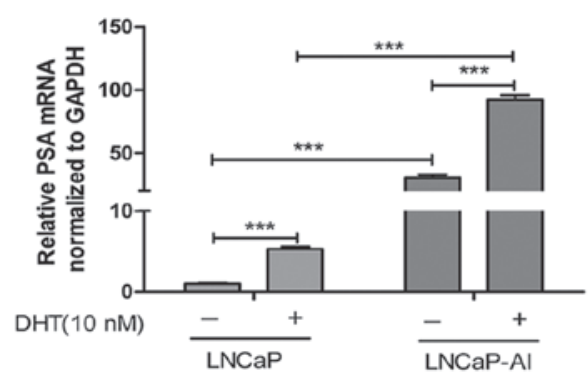

C

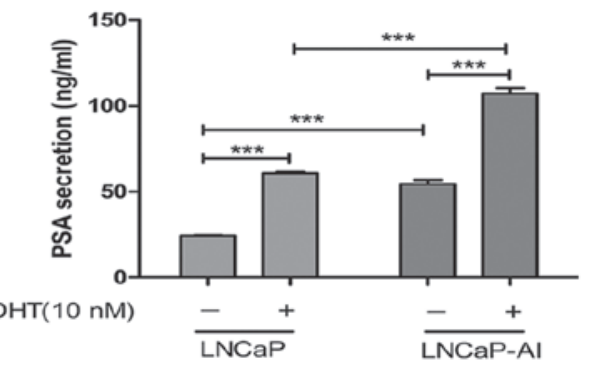

Figure 2. Androgen receptor (AR) expression levels and transcriptional activity in LNCaP and LNCaP-AI cells. (A) LNCaP and LNCaP-AI cells cultured for 2 days in CS-FBS medium were treated with ethanol (vehicle) or $10 \mathrm{nM}$ dihydrotestosterone (DHT) and lysates were immunoblotted for AR; GAPDH was used as a loading control (final ethanol concentrations were identical at 0.01\%). (B) RT-qPCR analysis of PSA mRNA expression in LNCaP and LNCaP-AI cells following treatment with $10 \mathrm{nM}$ DHT normalized to GAPDH. (C) LNCaP and LNCaP-AI cells were treated with $10 \mathrm{nM}$ DHT for $24 \mathrm{~h}$; cell culture supernatants were collected and PSA secretion levels were measured by chemiluminescence immunoassay (CLIA). Bars represent the means \pm SD of 3 replicates; ${ }^{* * * *} \mathrm{p}<0.001$.

p21 is a cyclin-dependent kinase inhibitor; however, androgen deprivation (EtOH group) induced the upregulation of p27 and p21 in the LNCaP cells (Fig. 3C). Therefore, p21 may play different roles in mediating G1 arrest in the LNCaP-AI and LNCaP cells.
Assessment efficiency of shRNA lentiviral vector transfection. At 3 days after transfection, cells expressing GFP were photographed under an inverted fluorescence microscope (Fig. 4A and E). Moreover, a FACSCalibur flow cytometer was used to detect GFP expression to assess the effi- 
A

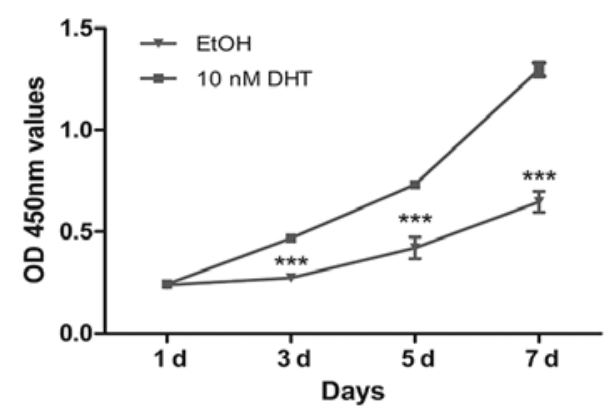

\section{LNCaP-Al cells}

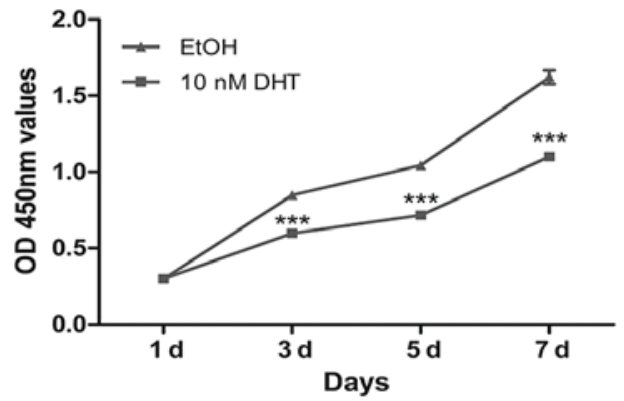

B
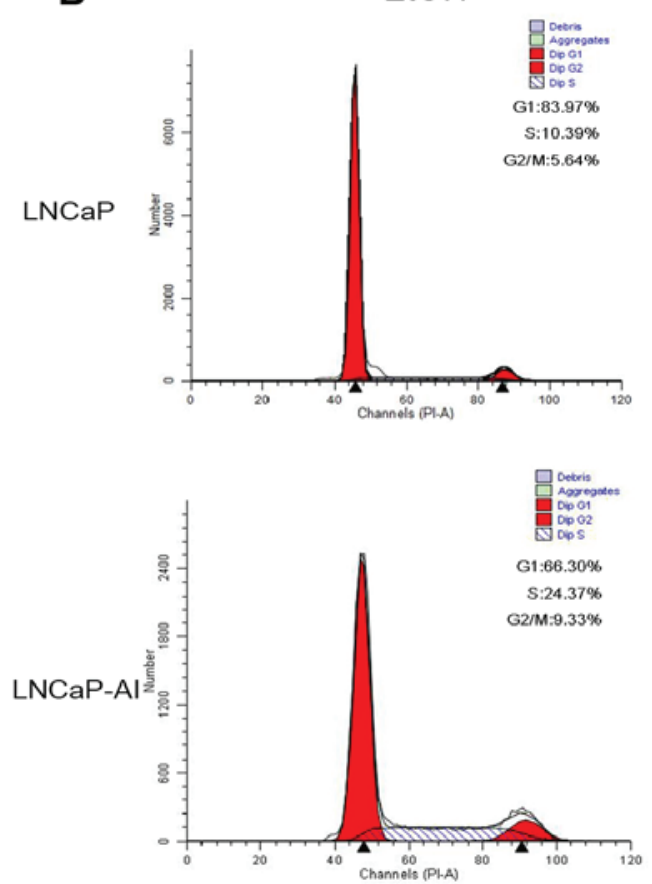

$10 \mathrm{nM} \mathrm{DHT}$
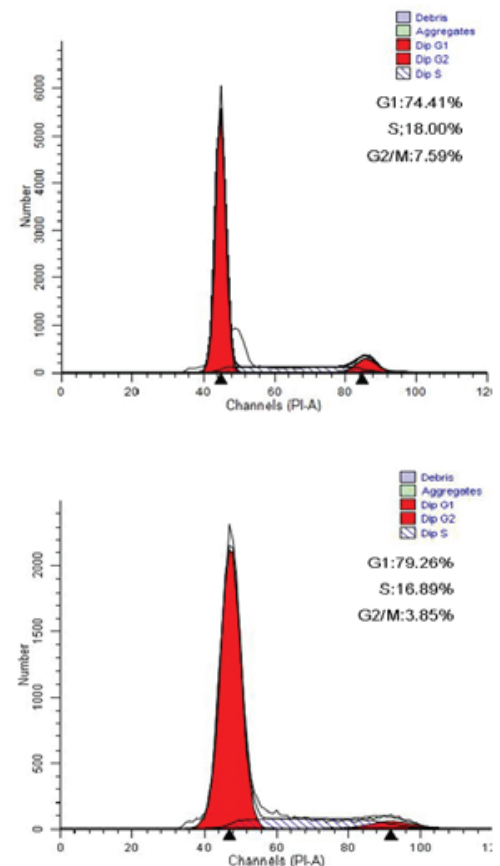

LNCaP cells

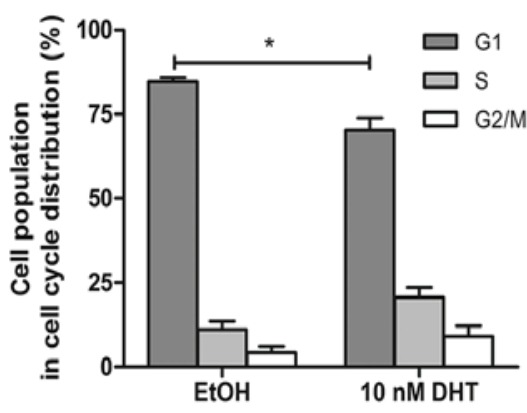

LNCaP-Al cells

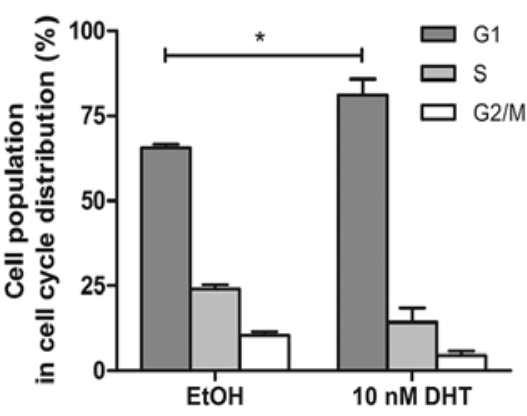

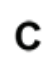
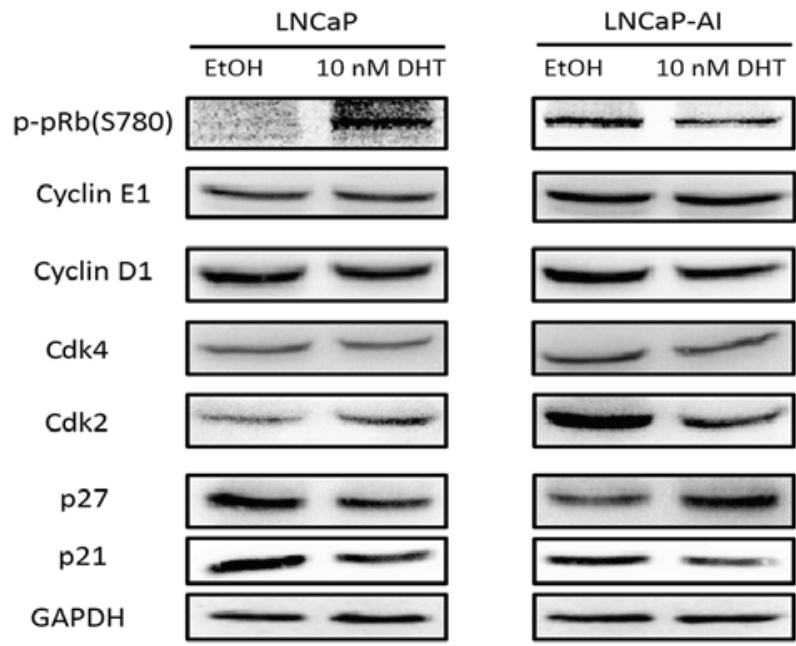

Figure 3. Androgen suppresses the proliferation of LNCaP-AI cells by leading to cell cycle arrest at the G1 phase. (A) LNCaP and LNCaP-AI cells were seeded in CS-FBS medium in 96-well plates and then treated with the vehicle or $10 \mathrm{nM}$ dihydrotestosterone (DHT) for different periods of time; cell proliferation measured by cell counting kit-8 (CCK-8) assay. (B) LNCaP and LNCaP-AI cells were cultured in CS-FBS medium in 6-well plates for 2 days and then treated with the vehicle or $10 \mathrm{nM}$ DHT for 2 days; the proportion of cells in the G1/G0, S and G2/M phases was detected by propidium iodide (PI) staining. (C) LNCaP and LNCap-AI cells were pretreated as in (B) and lysates were immunoblotted for G1 phase cell cycle-regulated proteins. Bars represent the means \pm SD of 3 replicates; ${ }^{*} \mathrm{p}<0.05$ and ${ }^{* * *} \mathrm{p}<0.001$.

ciency. The efficiency of all groups was $>80 \%$ (Fig. 4B and F). RT-qPCR was used to examine the mRNA level of AR after
shRNA lentiviral vector transfection. The results revealed that the AR levels in the LNCaP cells in the AR shRNA group 
A
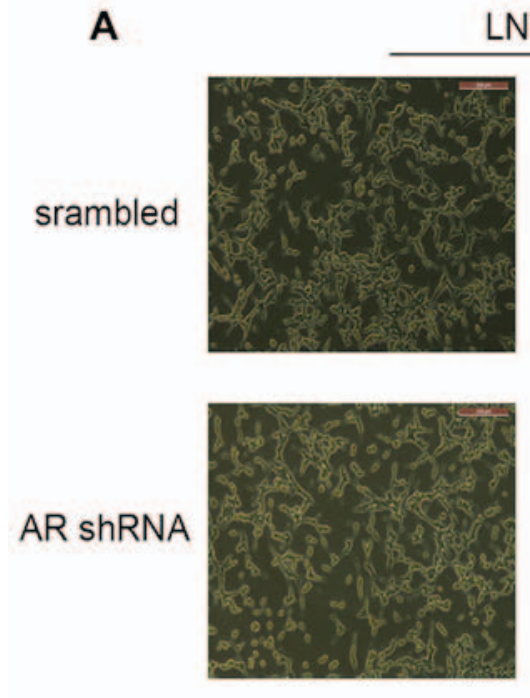

E

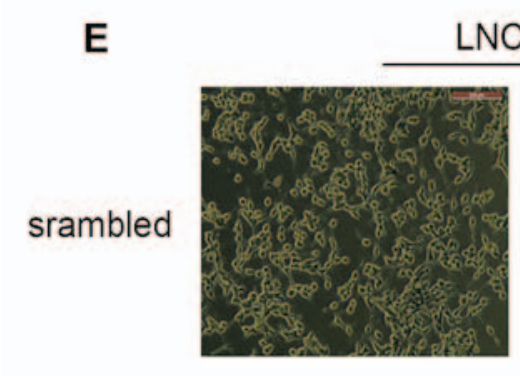

LNCaP-AI

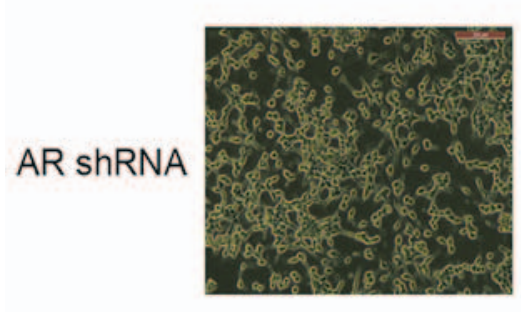

B
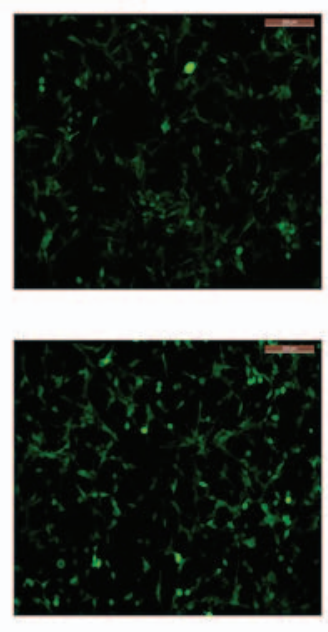

F
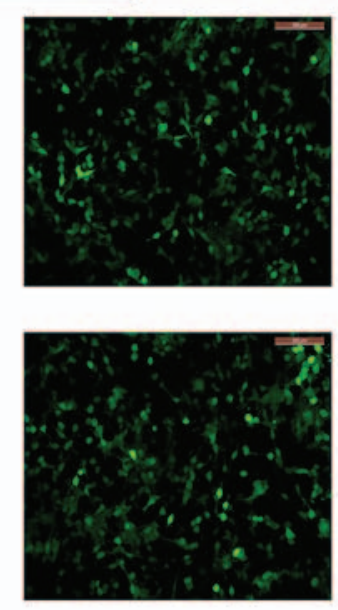
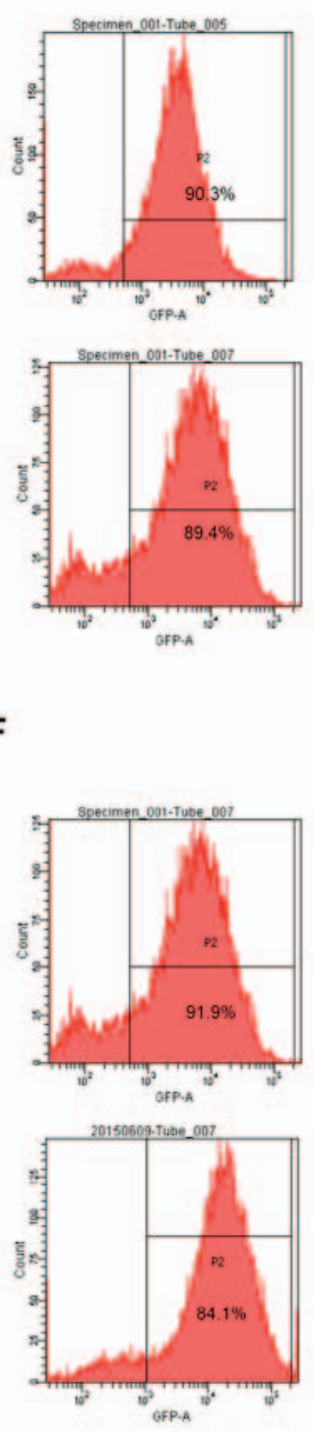

C

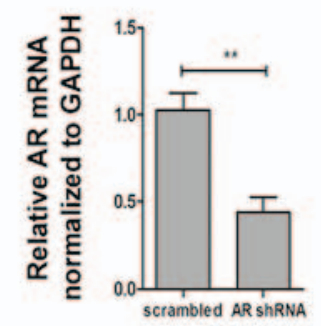

D

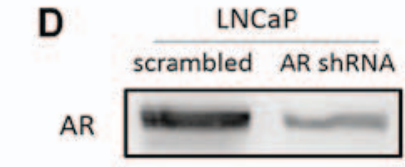

GAPDH

G

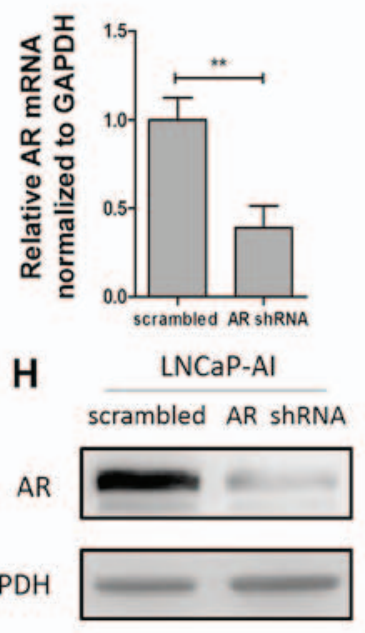

Figure 4. The efficiency of short hairpin RNA (shRNA) lentivirus transfection. (A and E) Androgen receptor (AR) and scrambled shRNA lentiviral vector transfection in LNCaP and LNCaP-AI cells; cells were photographed under an ordinary optical microscope or fluorescence microscope. (B and F) A FACSCalibur flow cytometer was used to detect green fluorescence protein (GFP) expression after lentiviral vector transfection. (C and G) The levels of AR mRNA after transfection was significantly decreased in the LNCaP and LNCaP-AI cells. (D and H) AR protein expression was significantly decreased by AR shRNA. Bars represent the means $\pm \mathrm{SD}$ of 3 replicates; ${ }^{* *} \mathrm{p}<0.01$.

decreased by $56.2 \%$ compared with those of the negative control group; the AR levels in the LNCaP-AI cells in the AR shRNA group decreased by $60.7 \%$ compared with the negative control group (Fig. 4C and G). As expected, the AR protein levels were also significantly decreased by transfection with AR shRNA lentiviral vector in the LNCaP and LNCaP-AI cells (Fig. 4D and H).

$A R$ remains a critical factor in the LNCAP-AI cells for cell cycle regulation and PSA secretion. To explore whether AR plays an important role in LNCaP-AI cells, the cells were subjected to AR shRNA or scrambled lentiviral particles transfection. CCK-8 assay, flow cytometry and CLIA were used to analyze cell proliferation, cell cycle distribution, the apoptotic rate and PSA secretion. The results revealed that AR silencing inhibited LNCaP-AI and LNCaP cell growth by inducing cell cycle arrest at the G1 phase (Fig. 5A and B); however, there was no appreciable increase in the apoptotic rate of the LNCaP-AI and LNCaP cells (Fig. 5C). Moreover, AR silencing notably decreased PSA secretion in the LNCaP-AI and LNCaP cells (Fig. 5D).

\section{Discussion}

Androgen deprivation therapy (ADT, surgical or medical castration) remains the mainstay treatment for all patients with $\mathrm{PCa}$ with metastatic disease $(15,16)$. However, the effects of these therapies are temporary, as within a median of 18-24 months, the disease ultimately progresses to an androgen-independent stage that is resistant to androgen ablation treatment, and is referred to as CRPC $(4,17)$. Therefore, to mimic the progression of androgen-dependent PCa to androgen-independent $\mathrm{PCa}$ in clinical practice, we established a subline of LNCaP cells (defined as LNCaP-AI cells) by continuous cultured in 
A

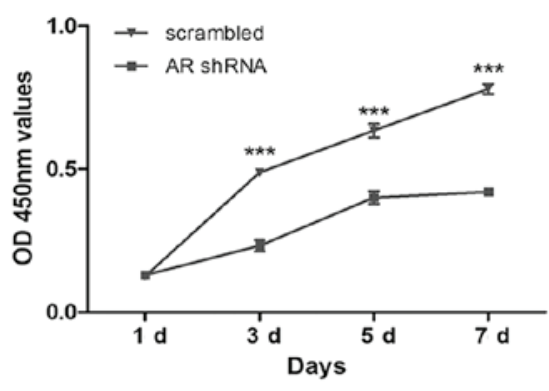

LNCaP-Al cells

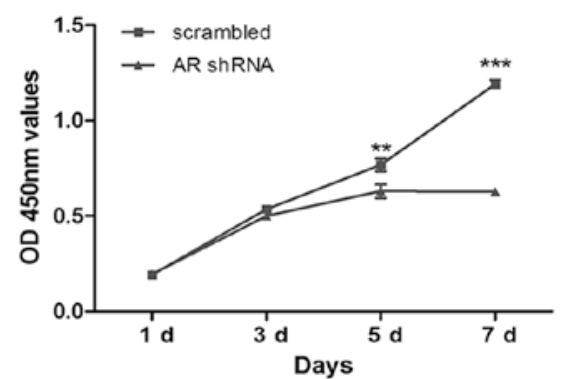

B
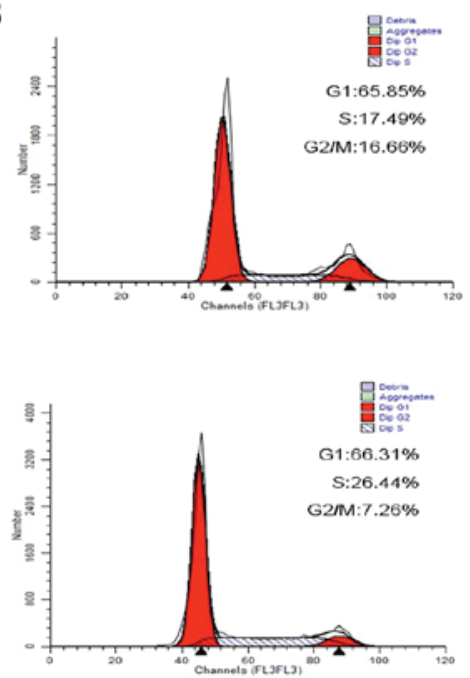

C
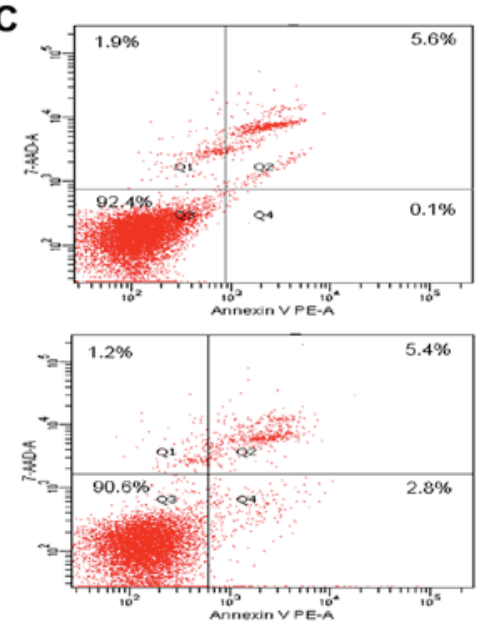

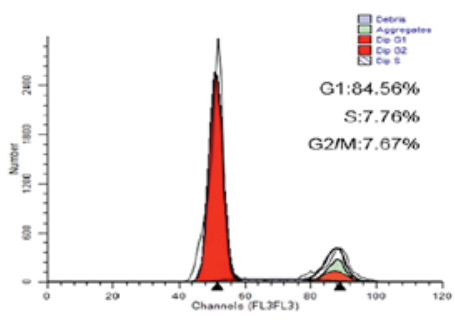

S: $7.76 \%$

$2 / \mathrm{M}: 7.67 \%$
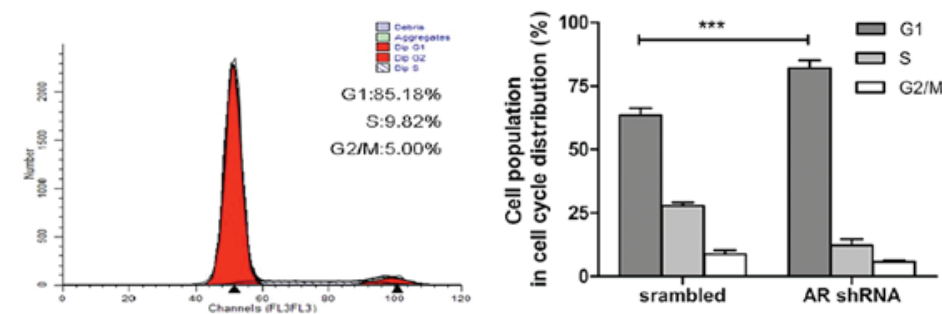

LNCaP cells
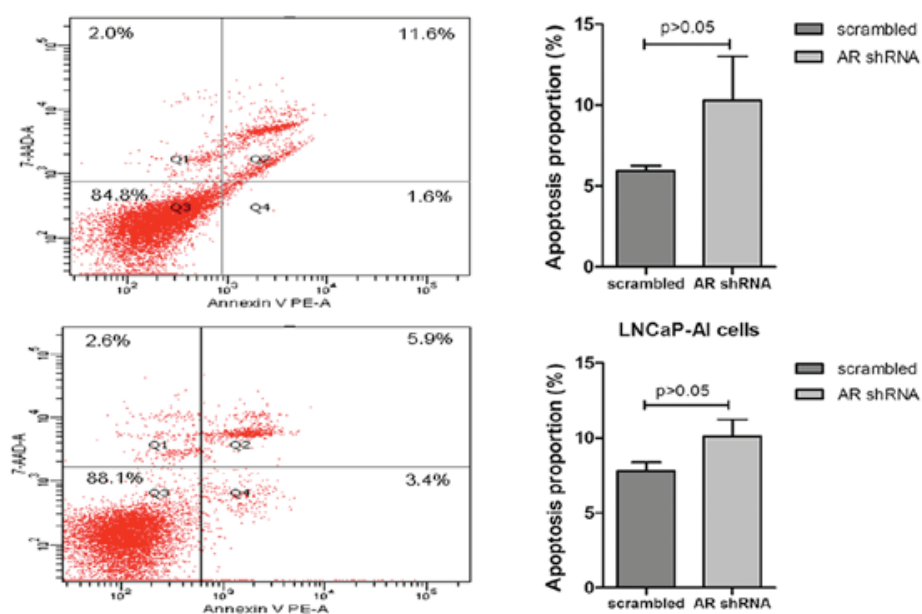

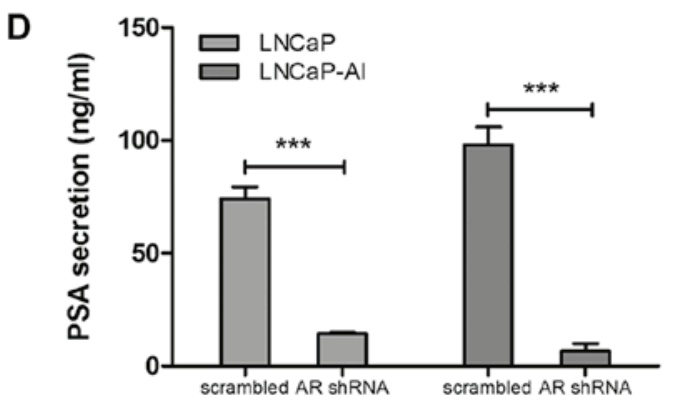

Figure 5. Androgen receptor (AR) short hairpin RNA (shRNA) suppresses LNCaP and LNCaP-AI cell growth by inducing G1 phase arrest, and reduces PSA secretion. (A) AR shRNA inhibited LNCaP and LNCaP-AI cell proliferation compared with the scrambled shRNA group. (B) AR shRNA increased the proportion of $\mathrm{LNCaP}$ and LNCaP-AI cells in the G1/G0 phase and decreased the proportion of those in the S phase compared with the scrambled group. (C) AR shRNA had no influence on the apoptosis of LNCaP and LNCaP-AI cells. (D) AR shRNA significantly reduced PSA secretion compared with the scrambled group in the LNCaP and LNCaP-AI cells using chemiluminescence immunoassay (CLIA). Bars represent the means \pm SD of 3 replicates; ${ }^{*} p<0.05$ and ${ }^{* * * *} \mathrm{p}<0.001$. 
the absence of androgen (13). In this study, we found that the LNCaP-AI cells represented a more aggressive phenotype compared with the LNCaP cells, as regards the cell proliferation rate, cell invasiveness, mesenchymal markers and PSA secretion. Androgen suppressed LNCaP-AI cell growth by inducing G1 phase arrest. Furthermore, we verified that AR remains a pivotal factor in the LNCaP-AI cells for cell cycle governance and PSA secretion.

To date, a number of studies have reported about androgenindependent LNCaP cells, and have demonstrated that androgen-independent LNCaP cells are characterized by an increased rate of cell proliferation in androgen-depleted medium compared with LNCaP cells $(14,18,19)$, consistent with our observation. However, there are few studies which have examined the effects of ADT on EMT and the invasiveness of LNCaP-AI cells. Sun et al reported that androgen deprivation caused EMT in PCa in vivo, and partially reconstituted in vitro by treating the LNCaP cell line with short-term androgen deprivation (20). This evidence further solidifies our findings that long-term androgen deprivation renders the LNCaP-AI cells to acquire a mesenchymal phenotype and stronger invasiveness compared with the LNCaP cells. On the whole, the present data suggest that LNCaP-AI cells, a subline of LNCaP cells, have a more aggressive phenotype.

The aberrant AR re-activation in CRPC has been reported in a variety of studies, and the mechanisms involved include AR amplification, gain of function AR mutations, ligandindependent $\mathrm{AR}$ activation and the overexpression of $\mathrm{AR}$ co-factors (4,21-24). Kokontis et al revealed that LNCaP-AI cells expressed a higher level of AR compared with LNCaP cells, and androgen increased AR protein expression (25). However, Lu et al obtained an opposite result that LNCaP-AI and LNCaP cells expressed similar levels of AR protein, and independent of androgen stimulation (14) consistent with our finding. Thus, the underlying mechanisms of aberrant AR re-activation are complex in CRPC. Of note, whether androgen is absent or present, the levels of PSA expression and secretion in LNCaP-AI cells were significantly higher than those in the LNCaP cells (Fig. 2B and C). Furthermore, Chuu et al found that the serum PSA level in castrated mice bearing 104-R2 (an androgen-independent LNCaP cell line) tumors was 8-fold higher than that of intact mice 104-S (an androgen-dependent LNCaP cell line) tumors (19). This may indicate that the serum levels of PSA in CRPC are also significantly higher than in androgen-dependent PCa. In our study, as shown in Fig. 5D, there is overwhelming evidence to indicate that aberrant AR re-activation occurs in CRPC, and androgen induces PSA secretion in LNCaP-AI cells via the AR signaling pathway.

Androgens are thought to be essential for $\mathrm{LNCaP}$ cell growth and survival. Under conditions of androgen deprivation, we found that LNCaP cell growth was suppressed by arrest in the G1 phase $(14,26,27)$. However, the effects of androgen on LNCaP-AI cells remain controversial. Lu et al demonstrated that the growth of LNCaP-AI cells still progressed with androgen stimulation (14). By contrast, Kokontis et al emphasized that androgen suppressed LNCaP-AI cell proliferation via the inhibition of Cdk2, Cyclin A and Skp2, and an increase in $\mathrm{p} 27$ protein accumulation, giving rise to cell cycle arrest at the $\mathrm{G} 1$ phase $(25,28)$. Our study showed that androgen led to $\mathrm{pRb}$-dependent $\mathrm{G} 1$ phase LNCaP-AI cell cycle arrest through the upregulation of p27, and the downregulation of $\mathrm{p} 21$ and $\mathrm{Cdk} 2$, ultimately resulting in the loss of $\mathrm{Rb}$ phosphorylation/inactivation. This is in agreement with the fact that p27 is a cyclin-dependent kinase inhibitor that binds to and prevents the activation of cyclin E-Cdk2 or cyclin D-Cdk4 complexes, and thus blocks cell cycle progression at G1 (29). Surprisingly, this observation is contrary to the role of p21 as a cyclin-dependent kinase inhibitor (30), and it is totally consistent with p21 as a positive regulator of cyclin-dependent kinase activity by promoting the formation, activation and nuclear enrichment of Cdk4/6-cyclin D complexes (31-34). Therefore, p21 may play a role as a positive regulator to promote $\mathrm{G} 1-\mathrm{S}$ transition in the LNCaP-AI cells in contrast to the LNCaP cells. Taken together, androgen exerts its suppressive effects on LNCaP-AI cell growth via the upregulation of p27 and the downregulation of p21 to inhibit CDK activity and cause G1 cell cycle arrest.

In order to further examine the effects of AR on LNCaP-AI cells, we designed AR-targeted shRNAs and used these to infect the LNCaP-AI and LNCaP cells. Not surprisingly, it appeared that the AR shRNA-transfected cells grew at a prominently slower rate compared with the scrambled shRNAtransfected cells; this was observed for both the LNCaP-AI and LNCaP cells. Our results are in accordance with those of other studies, which have reported that $\mathrm{AR}$ remains a critical factor for androgen-independent PCa cells (35-37). In general, AR silencing suppressed androgen-dependent $\mathrm{PCa}$ growth via a block of the G1-S transition (38). Thus, in this study, we investigated the underlying mechanisms through which AR inhibits the proliferation of LNCap-AI cells. We found that AR played a similar role in governing the cell cycle in both the LNCaP-AI and LNCaP cells; that is, AR silencing elicited G1 cell cycle arrest. In addition, AR silencing had no significant effect on the apoptosis of both LNCaP-AI and LNCaP cells, which was contradictory to the findings of previous studies showing that AR silencing leads to PCa cell death in vitro $(36,39,40)$. Nonetheless, Yuan et al demonstrated that AR downregulation did not result in significant apoptotic cell death in CWR22R3 cells (41). Perhpaps the reduction of AR protein abundance by shRNA lentivral infection was insufficient to trigger cell apoptosis. However, AR silencing decreased PSA secretion in the LNCaP-AI and LNCaP cells.

In conclusion, our data demonstrate that LNCaP-AI cells, a subline of LNCaP cells, have a more aggressive phenotype. AR remains a crucial factor for cell growth and the transcriptional activity of LNCaP-AI cells under androgen-depleted conditions. Moreover, androgen suppressed LNCaP-AI cell growth via the upregulation of p27 and the downregulation of p21, and reduced CDK activity, causing cell cycle arrest in the G1 phase. Therefore, LNCaP-AI cells can be defined as an androgenindependent/AR-dependent cell line with a more aggressive phenotype compared to the LNCaP cells.

\section{Acknowledgements}

This study was supported by a grant from the National Natural Science Foundation of China (no. 81271917). We would like to thank the Clinical Research Center of the Second Affiliated Hospital of Zhejiang University School of Medicine for providing technological support. 


\section{References}

1. Attar RM, Takimoto CH and Gottardis MM: Castration-resistant prostate cancer: locking up the molecular escape routes. Clin Cancer Res 15: 3251-3255, 2009.

2. Sharifi N, Kawasaki BT, Hurt EM and Farrar WL: Stem cells in prostate cancer: Resolving the castrate-resistant conundrum and implications for hormonal therapy. Cancer Biol Ther 5: 901-906, 2006.

3. Damber JE and Aus G: Prostate cancer. Lancet 371: 1710-1721, 2008.

4. Feldman BJ and Feldman D: The development of androgenindependent prostate cancer. Nat Rev Cancer 1: 34-45, 2001.

5. Ryan CJ, Smith A, Lal P, Satagopan J, Reuter V, Scardino P, Gerald W and Scher HI: Persistent prostate-specific antigen expression after neoadjuvant androgen depletion: An early predictor of relapse or incomplete androgen suppression. Urology 68: 834-839, 2006.

6. Tan MH, Li J, Xu HE, Melcher K and Yong EL: Androgen receptor: structure, role in prostate cancer and drug discovery. Acta Pharmacol Sin 36: 3-23, 2015

7. Heinlein CA and Chang C: Androgen receptor in prostate cancer. Endocr Rev 25: 276-308, 2004.

8. Tsihlias J, Zhang W, Bhattacharya N, Flanagan M, Klotz L and Slingerland J: Involvement of p27Kip1 in G1 arrest by high dose 5 alpha-dihydrotestosterone in $\mathrm{LNCaP}$ human prostate cancer cells. Oncogene 19: 670-679, 2000.

9. Hofman K, Swinnen JV, Verhoeven G and Heyns W: E2F activity is biphasically regulated by androgens in LNCaP cells. Biochem Biophys Res Commun 283: 97-101, 2001

10. Akakura K, Bruchovsky N, Goldenberg SL, Rennie PS, Buckley AR and Sullivan LD: Effects of intermittent androgen suppression on androgen-dependent tumors. Apoptosis and serum prostate-specific antigen. Cancer 71: 2782-2790, 1993.

11. Sciarra A, Abrahamsson PA, Brausi M, Galsky M, Mottet N Sartor O, Tammela TL and Calais da Silva F: Intermittent androgen-deprivation therapy in prostate cancer: A critical review focused on phase 3 trials. Eur Urol 64: 722-730, 2013.

12. Horoszewicz JS, Leong SS, Chu TM, Wajsman ZL, Friedman M, Papsidero L, Kim U, Chai LS, Kakati S, Arya SK, et al: The LNCaP cell line - a new model for studies on human prostatic carcinoma. Prog Clin Biol Res 37: 115-132, 1980.

13. Xu G, Wu J, Zhou L, Chen B, Sun Z, Zhao F and Tao Z: Characterization of the small RNA transcriptomes of androgen dependent and independent prostate cancer cell line by deep sequencing. PLoS One 5: e15519, 2010.

14. Lu S, Tsai SY and Tsai MJ: Molecular mechanisms of androgenindependent growth of human prostate cancer LNCaP-AI cells. Endocrinology 140: 5054-5059, 1999.

15. Loblaw DA, Mendelson DS, Talcott JA, Virgo KS, Somerfield MR, Ben-Josef E, Middleton R, Porterfield H, Sharp SA, Smith TJ, et al; American Society of Clinical Oncology: American Society of Clinical Oncology recommendations for the initial hormonal management of androgen-sensitive metastatic, recurrent, o progressive prostate cancer. J Clin Oncol 22: 2927-2941, 2004.

16. Sowery RD, So AI and Gleave ME: Therapeutic options in advanced prostate cancer: Present and future. Curr Urol Rep 8: 53-59, 2007.

17. Debes JD and Tindall DJ: Mechanisms of androgen-refractory prostate cancer. N Engl J Med 351: 1488-1490, 2004.

18. Wang Q, Li W, Zhang Y, Yuan X, Xu K, Yu J, Chen Z, Beroukhim R, Wang $\mathrm{H}$, Lupien $\mathrm{M}$, et al: Androgen receptor regulates a distinct transcription program in androgen-independent prostate cancer. Cell 138: 245-256, 2009.

19. Chuu CP, Kokontis JM, Hiipakka RA, Fukuchi J, Lin HP, Lin CY, Huo C, Su LC and Liao S: Androgen suppresses proliferation of castration-resistant LNCaP 104-R2 prostate cancer cells through androgen receptor, Skp2, and c-Myc. Cancer Sci 102: 2022-2028, 2011.

20. Sun Y, Wang BE, Leong KG, Yue P, Li L, Jhunjhunwala $S$, Chen D, Seo K, Modrusan Z, Gao WQ, et al: Androgen deprivation causes epithelial-mesenchymal transition in the prostate: Implications for androgen-deprivation therapy. Cancer Res 72 527-536, 2012

21. Chen CD, Welsbie DS, Tran C, Baek SH, Chen R, Vessella R, Rosenfeld MG and Sawyers CL: Molecular determinants of resistance to antiandrogen therapy. Nat Med 10: 33-39, 2004
22. Scher HI, Buchanan G, Gerald W, Butler LM and Tilley WD Targeting the androgen receptor: Improving outcomes for castration-resistant prostate cancer. Endocr Relat Cancer 11: 459-476, 2004

23. Scher HI and Sawyers CL: Biology of progressive, castrationresistant prostate cancer: Directed therapies targeting the androgen-receptor signaling axis. J Clin Oncol 23: 8253-8261, 2005.

24. Lonergan PE and Tindall DJ: Androgen receptor signaling in prostate cancer development and progression. J Carcinog 10:20, 2011.

25. Kokontis JM, Hay N and Liao S: Progression of LNCaP prostate tumor cells during androgen deprivation: Hormoneindependent growth, repression of proliferation by androgen, and role for p27Kip1 in androgen-induced cell cycle arrest. Mol Endocrinol 12: 941-953, 1998.

26. Knudsen KE, Arden KC and Cavenee WK: Multiple G1 regulatory elements control the androgen-dependent proliferation of prostatic carcinoma cells. J Biol Chem 273: 20213-20222, 1998.

27. Xu Y, Chen SY, Ross KN and Balk SP: Androgens induce prostate cancer cell proliferation through mammalian target of rapamycin activation and post-transcriptional increases in cyclin D proteins. Cancer Res 66: 7783-7792, 2006.

28. Kokontis JM, Lin HP, Jiang SS, Lin CY, Fukuchi J, Hiipakka RA, Chung CJ, Chan TM, Liao S, Chang CH, et al: Androgen suppresses the proliferation of androgen receptor-positive castration-resistant prostate cancer cells via inhibition of Cdk2, CyclinA, and Skp2. PLoS One 9: e109170, 2014.

29. Vidal A and Koff A: Cell-cycle inhibitors: Three families united by a common cause. Gene 247: 1-15, 2000.

30. Gartel AL, Serfas MS and Tyner AL: p21 - negative regulator of the cell cycle. Proc Soc Exp Biol Med 213: 138-149, 1996.

31. LaBaer J, Garrett MD, Stevenson LF, Slingerland JM, Sandhu C, Chou HS, Fattaey A and Harlow E: New functional activities for the p21 family of CDK inhibitors. Genes Dev 11: 847-862, 1997.

32. Sherr CJ and Roberts JM: CDK inhibitors: Positive and negative regulators of G1-phase progression. Genes Dev 13: 1501-1512, 1999.

33. Cheng M, Olivier P, Diehl JA, Fero M, Roussel MF, Roberts JM and Sherr CJ: The p21(Cip1) and p27(Kip1) CDK 'inhibitors' are essential activators of cyclin D-dependent kinases in murine fibroblasts. EMBO J 18: 1571-1583, 1999.

34. Alt JR, Gladden AB and Dieh1 JA: p21(Cip1) promotes cyclin D1 nuclear accumulation via direct inhibition of nuclear export. J Biol Chem 277: 8517-8523, 2002.

35. Zegarra-Moro OL, Schmidt LJ, Huang H and Tindall DJ: Disruption of androgen receptor function inhibits proliferation of androgen-refractory prostate cancer cells. Cancer Res 62: 1008-1013, 2002

36. Li TH, Zhao H, Peng Y, Beliakoff J, Brooks JD and Sun Z: A promoting role of androgen receptor in androgen-sensitive and -insensitive prostate cancer cells. Nucleic Acids Res 35: 2767-2776, 2007.

37. Snoek R, Cheng H, Margiotti K, Wafa LA, Wong CA, Wong EC, Fazli L, Nelson CC, Gleave ME and Rennie PS: In vivo knockdown of the androgen receptor results in growth inhibition and regression of well-established, castration-resistant prostate tumors. Clin Cancer Res 15: 39-47, 2009.

38. Comstock CE and Knudsen KE: The complex role of AR signaling after cytotoxic insult: Implications for cell-cycle-based chemotherapeutics. Cell Cycle 6: 1307-1313, 2007.

39. Liao X, Tang S, Thrasher JB, Griebling TL and Li B: Small-interfering RNA-induced androgen receptor silencing leads to apoptotic cell death in prostate cancer. Mol Cancer Ther 4: 505-515, 2005.

40. Yang Q, Fung KM, Day WV, Kropp BP and Lin HK: Androgen receptor signaling is required for androgen-sensitive human prostate cancer cell proliferation and survival. Cancer Cell Int 5: 8,2005

41. Yuan X, Li T, Wang H, Zhang T, Barua M, Borgesi RA, Bubley GJ, $\mathrm{Lu}$ ML and Balk SP: Androgen receptor remains critical for cell-cycle progression in androgen-independent CWR22 prostate cancer cells. Am J Pathol 169: 682-696, 2006. 